\title{
Fratura segmentar de clavícula: relato de 2 casos
}

\section{Segmental clavicle fracture: 2 case reports}

Jonatas Brito de Alencar Netoํ․ Maria Luzete Costa Cavalcante ${ }^{2}$. Pedro Henrique Messias da Rocha ${ }^{2}$. Renackson Jordelino Garrido².

1 Instituto Doutor José Frota (IJF), Instituto Dr Romeu Krause, Fortaleza, Ceará, Brasil. 2 Universidade Federal do Ceará (UFC), Fortaleza, Ceará, Brasil.

\section{RESUMO}

A clavícula por sua posição anatômica superficial apresenta grande risco a fraturas. No entanto, o tipo segmentar de fratura é algo raro, encontrando-se apenas um caso relatado na literatura brasileira. Isso se deve a combinação única de forças, que ocorrem, principalmente, em traumas de alta energia. O objetivo desse trabalho é apresentar dois casos de pacientes atendidos em serviço de referência traumatológica com diagnóstico de fratura segmentar de clavícula.

Palavras-chave: Clavícula. Fixação de fratura. Ortopedia.

\section{ABSTRACT}

The clavicle due to its superficial anatomical position presents a great risk to fractures. However, the segmental type of fracture is rare, being only one case reported in the Brazilian literature. This is due to the unique combination of forces, which occur mainly in high energy traumas. The objective of this study is to present two cases of patients assisted in a traumatologic referral service with a diagnosis of segmental clavicle fracture.

Keywords: Clavicle. Fracture fixation. Orthopedics.

Autor correspondente: Jonatas Brito de Alencar Neto, Rua Joaquim Nabuco, 1850, Aldeota, Fortaleza, Ceará. CEP: 60125-120. Telefone: +55 88 99702-1797. E-mail: jonatasbrito19@hotmail.com

Conflito de interesses: Não há qualquer conflito de interesses por parte de qualquer um dos autores.

Recebido em: 24 Jun 2018; Revisado em: 20 Mar 2019; Aceito em: 14 Ago 2019. 


\section{INTRODUÇÃO}

As fraturas de clavícula correspondem cerca de 2,6\% a 4\% de todas as fraturas em adultos, ${ }^{1}$ e de $10 \%$ a $15 \%$ de todas as fraturas em crianças. ${ }^{2}$ Entre as fraturas da clavícula, $69 \%$ ocorrem no terço médio, $28 \%$ no terço distal e $3 \%$ no terço proximal. $^{3}$

Essas fraturas são mais comuns em homens (70\%) e ocorrem, predominantemente, durante a prática de esportes e em acidentes automobilísticos. A lesão óssea é geralmente causada por traumas diretos sobre o ombro.

Em contrapartida, as fraturas segmentares são muito raras, tendo poucos casos descritos na literatura. ${ }^{2-4}$ Esse tipo de lesão exige uma combinação única de forças. Dessa forma, necessitam de energia de trauma maior e grande envolvimento de músculos e ligamentos adjacentes para gerar forças de tração. ${ }^{5}$

$\mathrm{Na}$ grande maioria dos casos, as fraturas de clavícula tem um diagnóstico clínico fácil devido a sua posição anatômica superficial, o que facilita sua palpação. ${ }^{3}$ No entanto, é fundamental diagnosticar possíveis lesões associadas. Como as fraturas segmentares são geradas por traumas de alta energia é essencial buscar nesses pacientes outras fraturas ou lesões pulmonares. As injúrias relacionadas mais comuns são: fratura de arcos costais ipsilaterais, fratura da escápula e/ou da glenoide, fratura de úmero proximal e hemo/ pneumotórax. ${ }^{6}$

No que concerne ao tratamento desse tipo de lesão clavicular a literatura aponta para a abordagem cirúrgica, haja vista a perda de vascularização óssea que pode existir. ${ }^{2,3,6}$

O objetivo desse trabalho é relatar dois casos de pacientes atendidos em hospital de referência traumatológica com diagnóstico de fratura segmentar de clavícula; caracterizada por ser rara e com apenas uma descrição na literatura brasileira.

\section{RELATO DOS CASOS}

\section{Caso 1:}

Paciente masculino, 30 anos, vítima de acidente automobilístico (capotamento de carro). Foi atendido em hospital de referência traumatológica da cidade de Fortaleza. Após avaliação clínica detalhada, paciente apresentou trauma cranioencefálico grave, sendo necessário tratamento cirúrgico da lesão. Após liberação clínica neurológica o paciente foi liberado para acompanhamento traumatológico.

Ao exame físico paciente apresentava dor, edema e deformidade em região clavicular direita. Além disso, evidenciou-se equimose (Figura 1). Não apresentou déficit neurovascular na cintura escapular.

Ao exame radiológico, o paciente apresentava fratura segmentar de clavícula direita nos terços médio e distal. As fraturas foram classificadas (classificação de Allman ${ }^{7}$ ) como: grupo 1 (terço médio) e grupo 2 (terço distal), sendo tipo 2B de Craig $^{8}$ (Figura 2).

Figura 1. Imagem ilustrando região de equimose em clavícula direita.

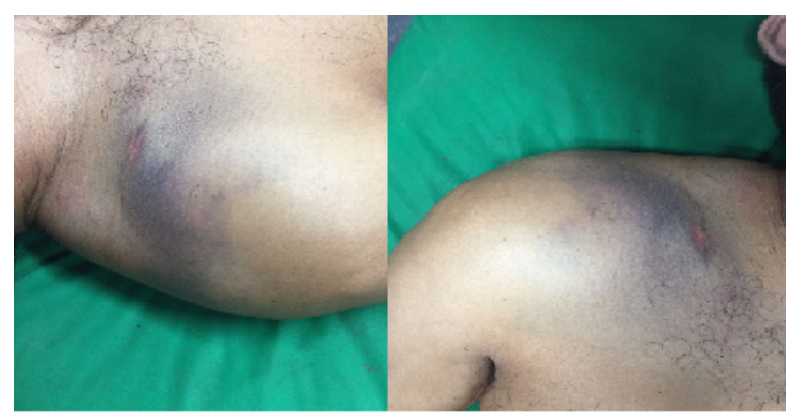

Figura 2. Radiografia anteroposterior de tórax evidenciando fratura de terço médio e distal de clavícula direita.

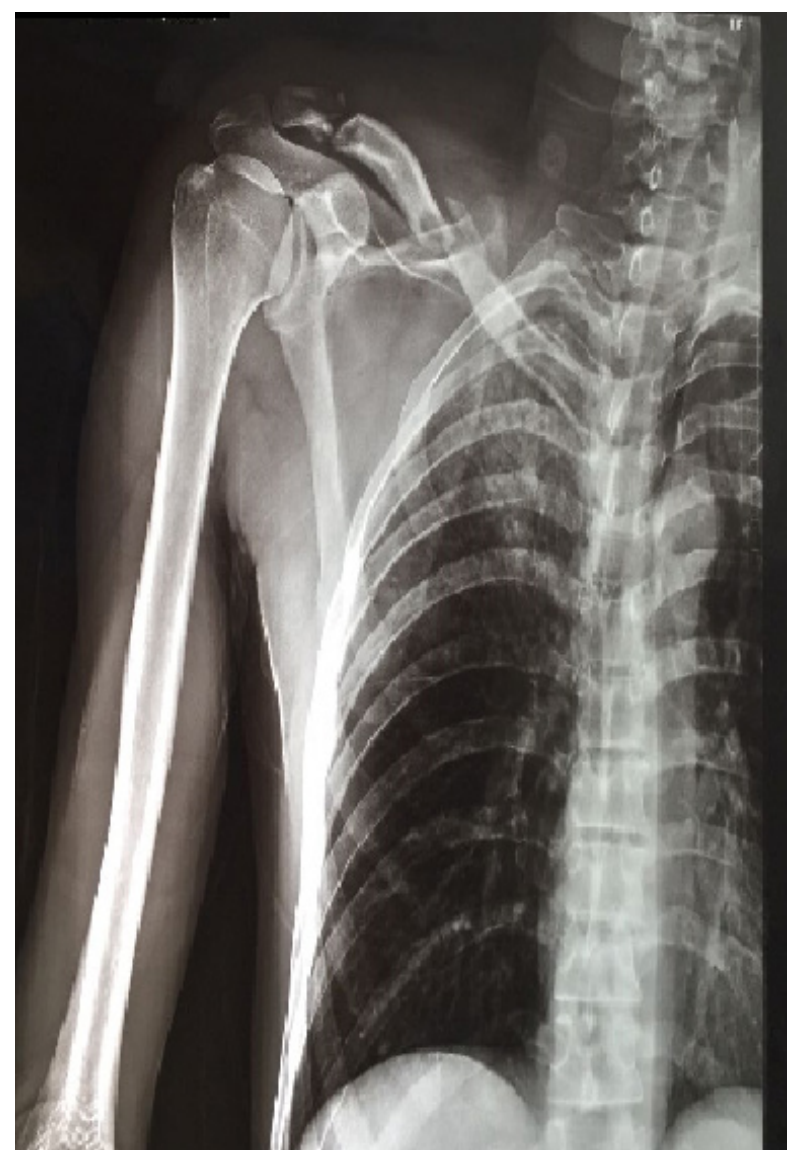

Para se definir o tratamento foram levados em conta aspectos importantes do paciente, como a energia do trauma, a idade do paciente e a dependência da utilização do membro nas atividades laborais. Ademais, critérios radiográficos, como a fratura segmentar e desvio maior que dois centímetros do foco de fratura medial. Nessa perspectiva foi decidido realizar tratamento cirúrgico eletivo. 
O princípio da estabilidade absoluta foi escolhido para osteossíntese, sendo utilizado uma redução aberta com método da compressão interfragmentária. O material utilizado foi uma placa de reconstrução $3.5\left(\right.$ Ortocir $\left.^{\circledR}\right)$ com sete parafusos corticais. Para cirurgia, optou-se por bloqueio anestésico regional de plexo braquial associado à sedação. $\mathrm{O}$ paciente estava em decúbito dorsal horizontal. $\mathrm{O}$ acesso utilizado foi o acesso longitudinal à clavícula.

Foi realizado acompanhamento ambulatorial com 15, 30, 60 e 120 dias. Paciente utilizou tipoia pré-fabricada nos primeiros quinze dias. Iniciou fisioterapia motora com exercícios isométricos ativos, bem como ganho de arco de movimento de punho, cotovelo e ombro. Fratura apresentou consolidação radiográfica com 11 semanas. Após esse tempo, o paciente estava com arco de movimento do ombro preservado sem limitações.

\section{Caso 2:}

Paciente masculino, 19 anos, vítima de acidente de carro. Foi atendido em hospital de referência traumatológica da cidade de Fortaleza. Após avaliação clínica detalhada, paciente relatou fortes dores no pescoço e na cabeça. Foi solicitado tomografia computadorizada de crânio e radiografia em perfil da coluna cervical em que não se evidenciou lesões cerebrais ou qualquer outra lesão mais grave. Dessa forma, foi liberado para acompanhamento traumatológico.

Ao exame físico, apresentava dor, edema e deformidade em região clavicular direita. Não apresentou déficit neurovascular na cintura escapular pelo trauma na clavícula. Além disso, paciente referiu dor em região posterior de tórax direito.

Ao exame radiológico, o paciente apresentava fratura segmentar de clavícula direita nos terços médio e distal. As fraturas foram classificadas (classificação de Allman) como: grupo 1 (terço médio) e grupo 2 (terço distal), sendo tipo 2B de Craig (Figura 3). Além disso apresentava fratura de terceiro e quarto arcos costais a direita (Figura 4). Não se evidenciou hemotórax ou pneumotórax por conta da lesão torácica.

Figura 3. Radiografia de clavícula direita evidenciando fratura de terço médio e distal.

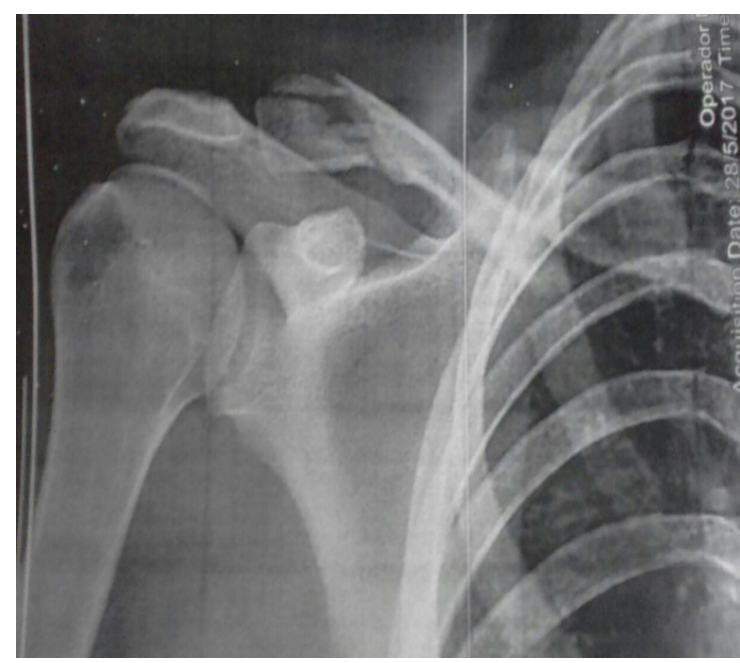

Figura 4. Radiografia de anteroposterior de tórax evidenciando fratura de terceiro e quarto arcos costais a direita (setas em vermelho).

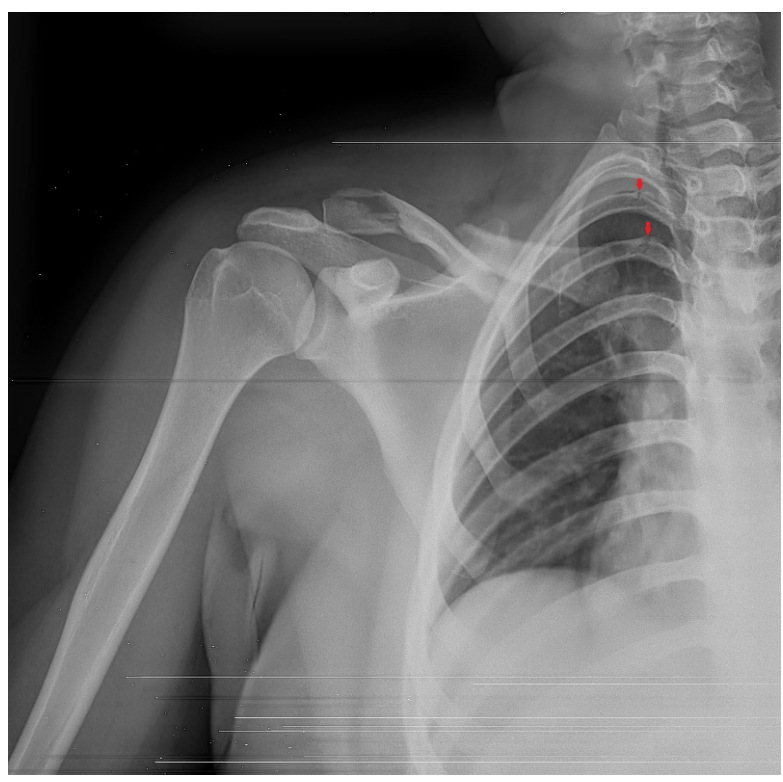

Após três dias de internamento hospitalar, o paciente recebeu alta, uma vez que não apresentava critérios cirúrgicos de fratura de clavícula (encurtamento $\geq 2 \mathrm{~cm}$, diástase $\geq 2 \mathrm{~cm}$, iminência de exposição óssea, lesão neurovascular ou fratura exposta $^{9}$ ) decidiu-se por tratamento não cirúrgico com uso de tipoia comercial tipo Velpeau.

Durante a alta, orientou-se exercícios isométricos de flexores e extensores do punho, isométricos de extensores e flexores do cotovelo e exercícios de ganho de arco de movimento de punho, cotovelo e ombro (até $90^{\circ}$ de abdução).

Foi realizado acompanhamento ambulatorial com 15, 30, 60 e 120 dias após a alta hospitalar. Após o primeiro mês, iniciou-se exercícios de ganho de arco de movimento (ADM) em ombro acima de $90^{\circ}$ de abdução. Aproximadamente dois meses após o trauma, o paciente ainda utilizava a tipoia e alegava perda de mobilidade do membro, conseguindo realizar abdução do braço direito até o ângulo de 90 graus. Foi solicitado fisioterapia agressiva para ganho de ADM do ombro direito.

Com 90 dias após a fratura, radiologicamente apresentava-se consolidada. Paciente apresentava ADM de ombro completo, bem como de cotovelo e punho. Iniciou-se fase final de fortalecimento muscular, retornando às atividades laborais normais após 120 dias do trauma.

\section{DISCUSSÃO}

As fraturas segmentares de clavícula são extremamente incomuns e pouco relatadas na literatura, ${ }^{5}$ tendo apenas um caso descrito na literatura brasileira. ${ }^{3}$

Os pacientes em questão apresentavam o mesmo tipo de fratura segmentar, ou seja, fraturas localizadas no terço médio e distal da clavícula. Isso vai ao encontro dos estudos encontrados sobre fratura segmentar, os quais relatam o mesmo tipo de lesão. ${ }^{3,5,10}$ 
O terço médio clavicular é a região clavicular que apresenta maior risco de fraturas, pois músculos e ligamentos se encontram apenas nos terços distal e lateral, o que acaba desprotegendo a região medial. ${ }^{1}$

O tratamento da fratura segmentar de clavícula é eminentemente cirúrgico, utilizando-se placa pré-moldada. Critérios com idade menor que 60 e maior que 15 anos; paciente que usa ativamente o membro; e paciente saudável são utilizados para a escolha do tratamento nesse tipo de fratura. ${ }^{6}$

Quando bem proposta, a cirurgia assegura um resultado satisfatório por diminuir o tempo de cicatrização óssea e a volta as atividades normais em menor prazo de tempo. ${ }^{11}$ Entretanto, necessita-se de maior número de casos descritos com acompanhamento para se ter mais dados acerca da indicação do melhor tratamento a depender do tipo de lesão.

\section{REFERÊNCIAS}

1. Kihlström C, Möller M, Lönn K, Wolf O. Clavicle fractures: epidemiology, classification and treatment of 2.422 fractures in the Swedish Fracture Register; an observational study. BMC Musculoskelet Disord. 2017;18(1):82.

2. Varelas N, Joosse P, Zermatten P. Operative treatment of an atypical segmental bipolar fracture of the clavicle. Arch Trauma Res. 2015;4(4):1-3.

3. Grossi EA. Fratura segmentar da clavícula. Rev Bras Ortop. 2011;5(6):733-5.

4. Daolagupu AK, Gogoi PJ, Mudiganty S. A rare case of segmental clavicle fracture in an adolescent. Case Rep Orthop. 2013;2013:1-2.

5. Yalizis MA, Hoy GA, Ek ET. A rare case of bipolar clavicle fracture. Case Rep Orthop. 2016;2016:3-5.

6. Mckee MD. Clavicle fractures. In: Rockwood CA, Green DP. Rockwood and green's fractures in adults. 8. ed. Philadelphia: Wolters Kluwer; 2015. p. 1427-74.
No entanto, por ter número limitado de casos na literatura, não há consenso sobre o manejo desses pacientes. Assim, abre-se espaço para outras formas de tratamento a depender do tipo de fratura. ${ }^{2}$

Um dos pacientes do presente estudo foi tratado de forma conservadora por não apresentar critérios radiológicos cirúrgicos. $^{9}$ Entretanto, apresentou maior período para ganho completo de ADM.

\section{CONCLUSÃO}

As fraturas segmentares de clavículas são raras, necessitando-se de exame radiológico adequado de todo o osso para diagnóstico preciso.

O tratamento não-cirúrgico pode ser executado com resultados razoáveis.

7. Allman FL Jr. Fractures and ligamentous injuries of the clavicle and its articulation. J Bone Joint Surg Am. 1967;49(4):774-84.

8. Craig EV. Fractures of the clavicle. In: Rockwood CA, Green DP, editors. Fractures in adults. 6. ed. Philadelphia: Lippincott Williams \& Wilkins; 2006. p. 1216-7.

9. Labronici PJ, Santos FC Filho, Reis TB, Pires RE, Mendes AF Junior, Kojima KE. Fraturas da diáfise da clavícula ainda são tratadas tradicionalmente, de forma não cirúrgica? Rev Bras Ortop. 2016;52(4):410-6.

10. Heywood R, Clasper J. An unusual ase of segmental clavicle fracture the literature, concurrent lateral. Army Med Corps. 2005;151(2);93-4.

11. Chaudhuri A, Datta S, Dugar N, Dutta S, Ghosh S, Gupta G, et al. Fracture clavicle: operative versus conservative management. Saudi J Sport Med. 2015;15(1):31.

\section{Como citar:}

Alencar JB Neto, Cavalcante ML, Rocha PH, Garrido RJ. Fratura segmentar de clavícula: relato de 2 casos. Rev Med UFC. 2020 janmar;60(1):51-54. 